\title{
Asociacionismo y participación voluntaria de los jóvenes españoles. Cambios y tendencias actuales
}

\author{
Associationism ans participation in volunteer activities among Spanish \\ young: changes and current trends
}

\author{
José Luis IZQUIETA ETULAIN \\ Universidad de Valladolid \\ izquieta@soc.uva.es \\ José Javier CALlEJo GonZÁLEZ \\ Universidad de Valladolid \\ javierc@soc.uva.es
}

Recibido: 19/05/2012

Revisado: 13/09/2012

Aceptado: 03/12/2012

Disponible on line: 16/04/2013

\section{Resumen}

Los estudios realizados en España sobre la participación social se centran preferentemente en el análisis de las asociaciones políticas tradicionales: partidos y sindicatos. Sin embargo la irrupción del voluntariado y el sustancial incremento de las asociaciones del Tercer Sector en los años noventa se interpreta habitualmente como una recuperación del interés de los jóvenes por la participación social y política; pero son escasos los análisis realizados sobre el alcance político y la proyección pública de la participación de los jóvenes en las asociaciones de voluntariado.

El presente trabajo se ocupa de este aspecto. Apoyados en los datos que aportan las encuestas realizadas sobre asociacionismo, y en los resultados obtenidos en diferentes investigaciones de carácter cualitativo sobre los jóvenes que participan en asociaciones altruistas, analizamos la expresión, los cambios y las disposiciones de su compromiso asociativo, contrastamos las interpretaciones realizadas sobre su participación asociativa y ofrecemos una explicación de su naturaleza, alcance y proyección social.

Palabras clave: participación social, jóvenes, voluntariado, ONG.

\begin{abstract}
The studies carried out in Spain on social involvement focus mainly on the analysis of traditional political associations: parties and labor unions. The growth in volunteering and the substantial increase of Third Sector associations in the nineties, however, are often interpreted as a recovery of young people's interest in social and political involvement; but there are few studies on the political effects and public scope of Spanish young people's associative involvement.

This work concerns this idea. Based on data derived from surveys about participation and on results obtained from a number of empirical studies about young people who get involved in altruistic associations, we analyze the expression, changes and the arrangements of their associative commitment and contrast the interpretations of their associative involvement to offer an explanation of their nature, effects and social scope.
\end{abstract}

Keywords: social involvement, young people, volunteering, NGO.

Referencia normalizada: Izquieta Etulain, J. L., y Callejo González, J. J. (2013): «Asociacionismo y participación voluntaria de los jóvenes españoles. Cambios y tendencias actuales». Cuadernos de Trabajo Social, 26(1): 159-170.

Sumario: Introducción. 1. Trayectoria y cambios del asociacionismo de los jóvenes españoles (1960-2010). 2. Participación y compromiso de los jóvenes españoles en las asociaciones altruistas. 3. Conclusiones. 4. Referencias bibliográficas.

\section{Introducción}

El interés y los debates académicos sobre la participación social y el asociacionismo han cobrado gran relevancia durante los últimos años en España. Dentro de la dinámica asociativa seguida por nuestra sociedad sobresalen 
distintos procesos cuyo alcance y proyección suscitan dudas e interrogantes con respecto a la mayor o menor implicación asociativa de los jóvenes. Los estudios empíricos confirman la expansión del volumen de las asociaciones cívicas y del voluntariado. ¿Puede o debe atribuirse este auge a la mayor preocupación asociativa de las nuevas generaciones? Los análisis recientes sobre el comportamiento político de los jóvenes españoles subrayan, así mismo, su creciente desafección y su alejamiento de los partidos políticos (Comas, 2009; Torcal y Montero, 2006); reconocen, no obstante, que ese desinterés se ve compensado por su participación en actividades políticas no convencionales (movimientos de protesta, organizaciones de voluntariado, uso de internet como instrumento de activación política, etc.) (Benedicto y Luque, 2006). ¿Comporta ese alejamiento de los partidos políticos su incorporación a otro tipo de asociaciones? ¿Cuál es el alcance político y la proyección pública de su participación en las asociaciones de voluntariado?

La consideración de estas cuestiones ha sido, hasta el momento, escasa y desigual. Los estudios sobre la participación social de los jóvenes españoles se centran preferentemente en el análisis de la participación en asociaciones políticas tradicionales (partidos políticos y sindicatos) y dejan en un segundo plano el estudio de las tendencias y las orientaciones que caracterizan su participación en otro tipo de asociaciones, como las de voluntariado. En el presente trabajo pretendemos indagar la trayectoria de su compromiso asociativo. Tratamos de identificar las motivaciones y las actitudes que definen su incorporación a las asociaciones altruistas. Deseamos aportar una explicación sobre los cambios y las tendencias de su compromiso e implicación social.

\section{Trayectoria y cambios del asociacionismo de los jóvenes españoles (1960-2010)}

El trayecto seguido por la participación asociativa de los jóvenes españoles en las últimas décadas puede delimitarse a través de los dos indicadores utilizados habitualmente en las encuestas nacionales realizadas a la juventud: la «tasa de asociacionismo» y las «preferencias asociativas». El primero de ellos viene dado por el porcentaje de jóvenes que en las encuestas afirman «pertenecer o formar parte de al- guna asociación, club, o grupo organizado». El segundo, recoge las tasas de pertenencia de los jóvenes a diferentes tipos de asociaciones. Ambos indicadores ofrecen una panorámica amplia y clarificadora de la trayectoria y de los cambios experimentados por la participación social de los jóvenes españoles durante las cuatro últimas décadas.

\subsection{Itinerarios y tendencias de la participa- ción asociativa de los jóvenes españoles}

Las constantes que caracterizan la trayectoria asociativa de los jóvenes españoles se aprecian, con cierta precisión, en las numerosas encuestas, informes y sondeos realizados en las últimas décadas sobre la juventud española. Los datos que recogen estos estudios se pueden agrupar en dos tablas que confirman, como hecho más significativo, la persistencia, con pequeñas variaciones, de las mismas tasas asociativas.

La primera de ellas abarca un periodo que va del año 1968 al 2007 y recoge datos relativos a jóvenes de 15 a 29 años. A pesar de que la formulación de las preguntas y el elenco de asociaciones que se han listado en las diferentes encuestas no han sido siempre los mismos, en la Tabla 1 se muestran unos resultados que se mueven entre el 31 por ciento en 1968 y el 36,9 por ciento en el año 2005 . De este intervalo queda excluida una de las encuestas realizada en 1995 y las realizadas en el 2004 y en el 2007, que ofrecen unos resultados claramente desviados. Si se obvian los resultados de estas tres encuestas, se aprecia un leve crecimiento (de 6 puntos porcentuales) de la tasa de asociacionismo a lo largo de los años 1990 respecto a 1968 (Ariño y Llopis, 2003, p. 174).

La segunda tabla recoge los porcentajes de jóvenes de 15 a 24 años que dicen «no pertenecer a ninguna asociación». La opción por esta segunda perspectiva se debe a que algunos informes, si bien ofrecen el porcentaje de jóvenes que dicen pertenecer a cada tipo de asociación, no ofrecen la tasa neta de asociación y en cambio ofrecen el porcentaje de los que dicen no pertenecer a ninguna (Tabla 2).

Al igual de lo que ocurre con la serie anterior, la formulación de las preguntas varía de unas encuestas a otras, pero también aquí, exceptuando la encuesta de 2005, el porcentaje de «no asociados» varía dentro de un intervalo 


\begin{tabular}{|c|c|c|c|c|c|}
\hline Pregunta & Año & Total & H & M & Difer. \\
\hline No consta & 1968 & 31,0 & 34 & 29 & 5 \\
\hline No consta & 1988 & 33,9 & 42,5 & 24,9 & 17,6 \\
\hline $\begin{array}{l}\text { ¿Perteneces actualmente a ...? (diferentes tipos de asociaciones } \\
\text { u organizaciones) ( por ciento de los que dicen pertenecer a alguna) }\end{array}$ & 1991 & 35,7 & 41,4 & 29,9 & 11,5 \\
\hline $\begin{array}{l}\text { ¿Perteneces a alguna de estas asociaciones, clubes } 0 \text { a algún partido } \\
\text { político o sindicato? por ciento Pertenecen a alguna }\end{array}$ & 1992 & 34,0 & 40,0 & 28,2 & 11,8 \\
\hline $\begin{array}{l}\text { ¿Formas parte de alguna asociación, organización, club o grupo organizado } \\
\text { de cualquier clase? } 1 \text {. No nuca he formado parte; } 2 \mathrm{Si} \text {, antes, pero ahora no; } \\
3 \mathrm{Si} \text {, formo parte de una o varias asociaciones (por ciento de los que } \\
\text { contestan afirmativamente a la tercera categoría) }\end{array}$ & $1995^{\mathrm{a}}$ & 36,0 & 39,0 & 32,0 & 7,0 \\
\hline $\begin{array}{l}\text { ¿Formas o formaste parte de alguna asociación, sociedad, club o grupo } \\
\text { organizado de cualquier clase bien sea cultural, deportivo, religioso, } \\
\text { ecologista, excursionista, sindical o de otro tipo? (por ciento Si ahora } \\
\text { estoy asociado) }\end{array}$ & $1995^{b}$ & 27,0 & 35,3 & 20,1 & 15,2 \\
\hline $\begin{array}{l}\text { De cada una de las siguientes asociaciones u organizaciones ¿Puedes } \\
\text { decirme si perteneces actualmente, si has pertenecido aunque ya no } \\
\text { pertenezcas, o si no has pertenecido nunca? (por ciento Pertenecen } \\
\text { actualmente) }\end{array}$ & 2000 & 37,0 & 44 & 31 & 13 \\
\hline $\begin{array}{l}\text { Voy a leerte una serie de asociaciones u organizaciones. } \\
\text { ¿De cada una de ellas puedes decirme si perteneces actualmente, has } \\
\text { pertenecido, aunque ahora ya no pertenezcas o si no has pertenecido nunca? } \\
\text { (por ciento de los que pertenecen actualmente a alguna) }\end{array}$ & 2003 & 37,5 & & & \\
\hline $\begin{array}{l}\text { De cada una de las siguientes asociaciones u organizaciones ¿Puedes } \\
\text { decirme si perteneces actualmente, si has pertenecido aunque ya no } \\
\text { pertenezcas, o si no has pertenecido nunca? (por ciento Pertenecen } \\
\text { actualmente) }\end{array}$ & 2004 & 25,0 & - & - & \\
\hline $\begin{array}{l}\text { Voy a leerte una serie de asociaciones u organizaciones. } \\
\text { ¿De cada una de ellas puedes decirme si perteneces actualmente, has } \\
\text { pertenecido, aunque ahora ya no pertenezcas o si no has pertenecido } \\
\text { nunca? (por ciento Pertenecen actualmente) }\end{array}$ & 2005 & 36,9 & 40,6 & 33,0 & 7,6 \\
\hline
\end{tabular}

Tabla 1. Evolución de la tasa de asociacionismo en los jóvenes (jóvenes 15-29 años).

Fuente:

1968: II Encuesta Nacional de Juventud, nov. 1968; 1931 casos; Datos procedentes del Informe Final (Anexo de Tablas) INJUVE, Documentos no publicados. Prieto Lacaci 1998, pág. 126.

1988: Encuesta Juventud en España 1988. Anexo estadístico, INJUVE. Prieto Lacaci (1993) en Informe Juventud en España 1992, pág. 181.

1991: Ecoconsulting (1991): Actitudes políticas de la juventud en España, INJUVE, pág. 30.

1992: Encuesta Juventud 1992. INJUVE, pág. 181.

1995a: EDIS (1995); La solidaridad de los jóvenes, INJUVE 1996. pág. 65.

1995b: Informe de Juventud en España 1996, INJUVE, pág. 365.

2000: Informe de Juventud en España 2000, INJUVE, pág. 634.

2002: CIS 2449, P.19 y P.20. Sondeo sobre la juventud española.

2003: CIS 2482, P.21 y P.22.

2004: Informe de Juventud en España 2004, INJUVE, pág. 576.

2005: CIS 2596, P.21 y P.22.

2007: CIS 2675, P.19 y P.25 (IJE 2008). 


\begin{tabular}{|c|c|c|c|c|}
\hline \multirow{2}{*}{ Año } & \multirow{2}{*}{ Pregunta } & \multicolumn{3}{|c|}{ Por ciento de no asociados } \\
\hline & & Total & Hom. & Muj. \\
\hline 1975 & $\begin{array}{l}\text { ¿Eres miembro de alguna asociación u organización? Si/no } \\
\text { (por ciento de los que constestan «no») }\end{array}$ & 73 & 68 & 79 \\
\hline 1984 & No consta & 67 & & \\
\hline $\begin{array}{l}1989 \\
1994 \\
1999\end{array}$ & $\begin{array}{l}\text { ¿A cuál de las siguientes cosas pertenece, si perteneces a } \\
\text { alguna? A ninguna }\end{array}$ & $\begin{array}{l}69 \\
69 \\
70\end{array}$ & 67 & 73 \\
\hline 2004 & $\begin{array}{l}\text { ¿Formas parte de alguna asociación, club juvenil o cualquier } \\
\text { otro grupo organizado ya sea de cultural, deportivo, religioso, } \\
\text { ecologista o de cualquier otro tipo? } \mathrm{Si} \text { / no (por ciento de los } \\
\text { que contestan «no») }\end{array}$ & 73 & 70 & 77 \\
\hline 2005 & $\begin{array}{l}\text { ¿A cuál de las siguientes asociaciones perteneces, si } \\
\text { perteneces a alguna? A ninguna }\end{array}$ & 81 & 79 & 83 \\
\hline 2010 & $\begin{array}{l}\text { ¿A cuál de las siguientes asociaciones perteneces, si } \\
\text { perteneces a alguna? A ninguna }\end{array}$ & 81 & 78,4 & 83,7 \\
\hline
\end{tabular}

Tabla 2. Evolución de la tasa de jóvenes no asociados (jóvenes 15-24 años).

Fuente:

1975: III Encuesta Nacional de Juventud. Revista del Instituto de la Juventud no 64, 1976, pág. 80.

1984: Juventud española 1984. Fundación Santa María, pág.74.

1989: Jóvenes españoles 89. Fundación Santa María, pág. 278.

1999: Jóvenes españoles 99. Fundación Santa María, pág., 241.

2002: Jóvenes 2000 y Religión. Fundación Santa María, pág. 145.

2004: Jóvenes y política. El compromiso con lo colectivo. INJUVE, pág. 68.

2005: Jóvenes 2005. Fundación Santa María, pág. 138.

2010: Jóvenes 2010. Fundación Santa María, pág. 80 (Cuestionario P9, pág. 369).

reducido, entre el 67 por ciento y el 81 por ciento. Sin embargo, en este caso se percibe una tendencia decreciente de la tasa de asociacionismo, sobre todo si se tienen en cuenta las encuestas realizadas en 2005 y 2010.

Los estudios, recogidos en estas dos tablas confirman, por tanto, que las tasas de asociacionismo de los jóvenes españoles han permanecido durante los últimos 30 años relativamente estables: entre el 30 y el 20 por ciento en el caso de los jóvenes de 15 a 24 años y en torno al 35 por ciento en el caso de los jóvenes de 15 a 29 años.

\subsection{Evolución y cambios en las opciones} asociativas de los jóvenes españoles

La continuidad o la escasa variación en las tasas netas de asociación no significan, sin embargo, que las pautas de participación de los jóvenes españoles hayan permanecido estables durante estos años. Cuando se analiza la evolución de las tasas de pertenencia a las distintas categorías asociativas se observan cambios sustanciales en el tipo de asociaciones que captan el interés de los jóvenes. El análisis de estos cambios presenta algunas dificultades derivadas de las diferencias que se producen entre unos estudios y otros en cuanto a los tipos y al número de asociaciones que se contemplan en los cuestionarios, lo que hace que los cambios no sean tan lineales y sencillos como desearíamos. A pesar de ello, el cambio en la elección de las asociaciones a las que se incorporan o en las que participan es inequívoco e incuestionable. El punto de partida en el que nos situamos para precisar este cambio es el año 1960. Retomamos para ello una encuesta realizada en un contexto social radicalmente distinto al actual caracterizado por la existencia de un régimen político autoritario, 


\begin{tabular}{|l|r|c|c|}
\hline & Total & Varones & Mujeres \\
\hline Culturales & 4,2 & 4,7 & 3,1 \\
\hline Musicales & 1,1 & 1 & 1,6 \\
\hline Cine clubes & 1,2 & 1 & 1,9 \\
\hline Organizaciones Juveniles & 12,7 & 16,1 & 3,1 \\
\hline Políticas & 5,7 & 4,8 & 8,6 \\
\hline Religiosas & 20 & 14,7 & 38 \\
\hline Deportivas & 12,3 & 13,7 & 7,7 \\
\hline De antiguos alumnos & 6,5 & 4,1 & 14,4 \\
\hline Profesionales & 0,7 & 0,5 & 1,4 \\
\hline Sindicales & 2,7 & 2,8 & 2,4 \\
\hline Cooperativas & 0,2 & 0,2 & 0,2 \\
\hline Excursionistas & 2,1 & 2,1 & 2,1 \\
\hline N & & $(1.316)$ & $(415)$ \\
\hline
\end{tabular}

Tabla 3. Concretamente de este tipo de asociaciones ¿a cuál o cuáles perteneces? Año 1960 (jóvenes 16-20 años).

Fuente: Encuesta sobre presupuestos mentales de la juventud española (1976). Revista del Instituto de la Juventud, 64, p. 221.

una cultura dominada por el catolicismo tradicional y unas estructuras económicas y sociales en proceso de transformación, pero todavía premodernas. La encuesta muestra los siguientes porcentajes de asociación.

La distribución en conjunto revela un claro predominio del llamado «modelo de asociacionismo juvenil tradicional». Este modelo se caracteriza por el encuadramiento de una parte de la población juvenil en organizaciones dirigidas por adultos con el propósito de contribuir a la socialización secundaria de los jóvenes, especialmente en el ámbito del ocio y del tiempo libre, de acuerdo a unas determinadas doctrinas filosóficas, religiosas o políticas que reflejaban el pluralismo reformista de la época (Prieto, 1985, p. 39).

Este tipo de asociacionismo entra en crisis en los años 1960, con la transformación social y política de la sociedad española. A partir de esa década surge un nuevo modelo de asocia- cionismo juvenil que da respuesta sobre todo a sus necesidades expresivas e instrumentales (Prieto Lacaci, 1998, pp. 24-27). La expansión y la consolidación de este modelo se constata en la distribución de las preferencias asociativas de los jóvenes que muestran las siguientes tablas en las que se recogen datos procedentes de encuestas sobre jóvenes de 15 a 24 años y de 15 a 29 años, realizadas, en su mayor parte, en las décadas de 1980, 1990 y 2000. En ellas aparecen también los datos de sendas encuestas pertenecientes a la etapa anterior, ofreciendo de este modo la posibilidad de percibir los cambios operados en el paso del modelo «tradicional» al nuevo o «moderno».

La comparación de los datos que aparecen en estas tablas con los de la encuesta de 1960 muestra el retroceso del asociacionismo religioso, dominante en el modelo tradicional, y el descenso del asociacionismo juvenil. Estos cambios reflejan la práctica desaparición del asociacionismo juvenil tradicional y su sustitución por otro tipo de asociaciones

El asociacionismo político presenta una trayectoria diferente. Parte en los años 1960 de unos porcentajes de afiliación importantes, en torno al 5 por ciento, debido probablemente, como se ha apuntado anteriormente, al carácter político de algunas organizaciones en las que se encuadraban los jóvenes; alcanza sus máximos históricos en los años de la transición política, 8 por ciento en 1977 (III Encuesta Nacional de Juventud, 1978, p. 159), y cae a finales de los años ochenta a unos niveles muy bajos, entre el 1 por ciento y el 2 por ciento. Este descenso se aprecia tanto en lo que se refiere a la participación en los partidos políticos como a la participación en las asociaciones reivindicativas (movimientos sociales) y de derechos humanos.

Por otra parte, el asociacionismo deportivo muestra una clara tendencia al alza. La participación en este tipo de asociaciones no solamente crece sino que se constituye además en la participación dominante entre los jóvenes, captando prácticamente el 50 por ciento de la pertenencia asociativa de los jóvenes.

Mención aparte hay que hacer del asociacionismo benéfico-asistencial (asociaciones del Tercer Sector o de voluntariado), modelo del que nos ocuparemos en el apartado siguiente. Este tipo de asociaciones presentan una 


\begin{tabular}{|c|c|c|c|c|c|c|c|c|}
\hline & $* 1975$ & 1984 & 1989 & 1994 & 1995 & 2004 & 2005 & 2010 \\
\hline Asociaciones y clubes deportivos & 8,8 & 17 & 8 & 14 & 12 & 12 & 5,6 & 6.5 \\
\hline $\begin{array}{l}\text { Sociedades locales (peñas, fiestas, } \\
\text { cofradías) }\end{array}$ & & & & & & & 2,7 & 2.8 \\
\hline Grupos educativos artísticos o culturales & 7,9 & 7 & 4 & 6 & 5,5 & 5 & 4 & 3.1 \\
\hline $\begin{array}{l}\text { Organizaciones y grupos juveniles } \\
\text { (scout, guías, clubes) }^{1}\end{array}$ & 7,1 & 5 & 5 & 5 & 6 & & 2,6 & 2,3 \\
\hline Asociaciones de tipo religioso ${ }^{2}$ & 3,6 & 6 & 4 & 4 & 3,5 & 2,7 & 2,5 & 1,6 \\
\hline Partidos Políticos & 1,5 & 1 & 1 & 1 & 0,8 & 1,3 & 1,1 & 0.8 \\
\hline Sindicatos & & & & & & 0,9 & 1,2 & 0,9 \\
\hline $\begin{array}{l}\text { Grupos ecologistas o de protección } \\
\text { de los animales }\end{array}$ & & 2 & 2 & 2 & 1,6 & 1,2 & 1,6 & 1,6 \\
\hline Mujer, feministas & & & & & & & 0,3 & 0,3 \\
\hline Antiglobalización & & & & & & & 0,2 & 0,1 \\
\hline $\begin{array}{l}\text { Organizaciones interesadas por los } \\
\text { derechos humanos }\end{array}$ & & 1 & 1 & 1 & 0,5 & 0,5 & 0,9 & 0.8 \\
\hline Ayuda el Tercer Mundo & & & & & & & 1,0 & 0,8 \\
\hline $\begin{array}{l}\text { Sociedades benéficas interesadas } \\
\text { por el bienestar de la gente }\end{array}$ & & 1 & 2 & 2 & 3 & 1,5 & 2,1 & 2,4 \\
\hline No pertenece a ninguna de ellas & 74 & 67 & 74 & 69 & 70 & 73 & 81 & 81 \\
\hline
\end{tabular}

Tabla 4. Tipos de asociaciones a las que pertenecen los jóvenes (15-24 años).

Fuente:

Para el año 1975: III encuesta nacional de la juventud. Para los años 1984, 1989, 1994 y 1998: Jóvenes españoles 99, pág. 243, Fundación Santa María.2004 Jóvenes y política, INJUVE pago. 682005 y 2010 Jóvenes españoles 2005 y 2010 Fundación Santa María pág. resp. 138 y 8.0

Nota: Por otra parte en la confección de la tabla se han excluido algunas categorías asociativas que sólo aparecen en una encuesta. Concretamente las asociaciones excursionistas, los club sociales o asociaciones recreativas que aparecen en 2004, ambas con un 2,3 por ciento de afiliación, las peñas futbolísticas que solo aparecen en 1994. con un 4 por ciento y las asociaciones cívicas que solo aparecen en 2004 con un 0,7 por ciento.

${ }^{1}$ En 1984 y 1989, «trabajo con grupos juveniles» (p. ej. scout, guías, clubes juveniles).

${ }^{2}$ En 1984, «iglesias u organizaciones religiosas».

evolución peculiar debido a que no se contemplan en las encuestas hasta los años 1980. Los primeros datos sobre pertenencia o implicación de los jóvenes españoles en estas asociaciones revelan tasas muy bajas, pero confirman su progresivo incremento: el 1 por ciento en 1984 y el 3 por ciento en 1998, como puede verse en la Tabla 4. Las encuestas del Instituto de la Juventud (INJUVE) no contemplan la participación en este tipo de asociaciones hasta la década de los años 1990. En ellas se aprecia que su pertenencia gira en torno al 3 por ciento o al 4 por ciento. Ahora bien, si en lugar de preguntar a los jóvenes por su pertenencia o no a una asociación benéfica u ONG, se les pregunta directamente por su participación en actividades de voluntariado las respuestas afirmativas se elevan hasta el 5 por 
José Luis Izquieta Etulain y José Javier Callejo González Asociacionismo y participación voluntaria de los jóvenes...

\begin{tabular}{|c|c|c|c|c|c|c|c|c|c|}
\hline & 1968 & 1988 & 1991 & 1992 & 1995 & 2000 & 2002 & 2003 & 2005 \\
\hline Pertenecen a alguna asociación & 31 & 34 & 36 & 34 & 36 & 37 & 37,5 & 38 & 37 \\
\hline Deportiva & 11,3 & 17,8 & 18 & 16,8 & 19 & 18 & 17,9 & 20,5 & 18,4 \\
\hline Cultural, educativa & 4,1 & 7,8 & 7 & 6,3 & 7 & 8 & 8,2 & 8,7 & 7,3 \\
\hline Musicales & 1,9 & & & & & & 4,7 & 4,8 & \\
\hline Juvenil & 3,5 & 2,8 & 4 & & & & & & \\
\hline Estudiantil & 3,1 & $\mathrm{~s} / \mathrm{d}$ & & 3,7 & 3 & & 6,3 & 6,2 & 5,7 \\
\hline Excursionista & 1,6 & & & & 5 & 5 & 3,8 & 4,3 & 3,9 \\
\hline Recreativa(a) & & 3,8 & 5 & 3,5 & 3 & & 6,4 & 6,5 & 5,5 \\
\hline Festiva(peñas, fallas) y cofradías & & & & & 12 & 12 & & & \\
\hline Religiosa & 9,4 & 4,8 & 5 & 4,7 & 9 & 5 & 5,8 & 5,4 & 4,7 \\
\hline Política & 1,6 & 1,9 & & 2,0 & 1 & 1 & 1,1 & 1,1 & 1,3 \\
\hline Sindical & 1,6 & 1,3 & 4 & 3,0 & 1 & 2 & 2,1 & 1,6 & 1,7 \\
\hline Cívica & & 1,4 & 5 & 1,6 & 2 & & 2,7 & 1,9 & 1,5 \\
\hline Feminista & & & & & 1 & & 0,1 & 0,1 & 0,3 \\
\hline Ecologista(b) & & 1,6 & 2 & 2,3 & 2 & 2 & 2,5 & 3 & \\
\hline Pacifista & & & & & 2 & 1 & 1 & 1,3 & 0,9 \\
\hline Benéfico/asistencial & & & & 1,3 & 4 & 4 & 4,5 & 3,6 & 3,4 \\
\hline Derechos Humanos & & & & & 1 & 2 & 1,4 & 1,5 & 2 \\
\hline Otras & & 1,3 & & 1,7 & 4 & & 0,9 & 0,2 & 1,7 \\
\hline $\mathrm{N}$ & & 5.249 & 2.012 & 5.000 & 1.200 & 6.492 & 1.490 & 1.457 & 1.433 \\
\hline
\end{tabular}

Tabla 5. Tipos de asociaciones a las que pertenecen los jóvenes (15-29 años) por ciento de los que dicen pertenecer a cada uno de los tipos de asociaciones citados.

Fuente:

Año 1988: Zárraga, J.L. (1989); Encuesta Juventud 1988, Anexo estadístico, Instituto de la Juventud.

Año 1991: Ecoconsulting (1991): Actitudes políticas de la juventud en España. Instituto de la Juventud. Año 1992: Navarro y Mateo (1993); Encuesta Juventud 1992.

Año 1995: EDIS (1995); La solidaridad de los jóvenes, Instituto de la Juventud 1996.

Año 1995 b: Martín Serrano, M (1996): Informe Juventud en España.

Año 2000: Informe Juventud en España 2000. INJUVE, pág. 635.

Año 2002: CIS 2449 P.19 Y P.20.

Año 2003: CIS 2482 P.21 Y P.22.

Año 2004: Informe de Juventud en España 2004, INJUVE, pág. 584.

El IJE-92 une las asociaciones recreativas y excursionistas.

El IJE- 92 engloba pacifistas y feministas.

Nota: En esta tabla no se han incluido los datos procedentes del Informe de Juventud del INJUVE, de 1996 y 2004 y 2010 porque ofrecen unas tasas de asociacionismo en los jóvenes inusualmente bajas, aunque han sido recogidos con la misma metodología que otros estudios contemplados en la tabla. 
ciento en 1994 y al 12 por ciento en 1995 (EDIS, 1995) y al 10 por ciento en 2006 (CIS, Estudio 2642, 2002). Esta evolución muestra el carácter novedoso de estas iniciativas en el panorama asociativo de los jóvenes españoles.

La presencia y los porcentajes significativos de afiliación que obtienen este tipo de asociaciones en los años noventa y primeros años de la década actual se corresponden con la expansión alcanzada por este tipo de asociaciones en esa época. Durante el trienio de 19871989 crecen al ritmo del 32 por ciento y en los años 1990-1992 se mantienen, mostrando una media anual de más de 10.000 asociaciones (Mota, 1999, p. 49). Entre 1999 y 2002 constituyó el 42 por ciento de las entidades asociativas inscritas actualmente en los registros de asociaciones (Izquieta, 2011, p. 150).

Dentro del asociacionismo pueden distinguirse, siguiendo la terminología de Ariño y Llopis, entre un asociacionismo autoorientado dedicado a la satisfacción de necesidades instrumentales o expresivas de sus socios (asociaciones de tipo cultural, deportivo o festivo) y un asociacionismo heteroorientado, en el que se incluyen las asociaciones reivindicativas (movimientos sociales y de derechos humanos) y las benéfico asistenciales (de voluntariado) (Ariño y Llopis 2003, p.176).

Los datos recogidos en este apartado revelan que el asociacionismo autoorientado es mayoritario y da muestras de crecimiento. Por el contrario, el asociacionismo heteroorientado tiene, a partir de los años 1980, un peso reducido en las preferencias asociativas de los jóvenes y no muestra signos de crecimiento, a excepción del asociacionismo que se plantea en las asociaciones benéfico-asistenciales (asociaciones del Tercer Sector o de voluntariado).

\section{Participación y compromiso de los jóve- nes españoles en las asociaciones altruistas}

La participación de los jóvenes en las asociaciones altruistas suscita, no obstante, algunos interrogantes sobre el alcance político de la susodicha y sobre si su implicación puede considerarse una alternativa o una revitalización del compromiso cívico. Para despejar estas dudas trataremos de identificar las motivaciones que impulsan su compromiso en este tipo de asociaciones y analizaremos sus actitudes y disposiciones.

\subsection{Motivaciones de la participación de los jóvenes españoles en las asociaciones al- truistas}

Dentro de las investigaciones realizadas en los últimos años sobre los jóvenes que colaboran en las asociaciones altruistas sobresale la efectuada en 1995 por EDIS. Este estudio cuantitativo confirma que la participación de los jóvenes españoles en asociaciones de voluntariado se origina por razones diferentes a las que predominan en la participación política. En la Tabla 4 se aprecia que la mayoría de los jóvenes, el 74 por ciento, y 8 de cada 10 de los que ya ejercen como voluntarios se muestran «muy o bastante de acuerdo» con la necesidad de «ver los frutos de la colaboración». Este rasgo distingue al compromiso voluntario del activísimo político, pues en éste rara vez se llegan a contemplar los frutos del esfuerzo realizado, debido fundamentalmente al carácter general y universal de los objetivos que persigue.

El estudio EDIS muestra que el compromiso de los jóvenes en las asociaciones de voluntariado se proyecta sobre los demás, pero busca o persigue también la obtención de algún tipo de compensación o beneficio personal. Tal como puede verse en la Tabla 4, el 75 por ciento considera que el voluntariado debe aportar beneficios no económicos, lógicamente, pero sí relacionados con la realización personal. La tabla revela que el voluntariado es, o debe ser un espacio de relación y de comunicación con los otros: el 74 por ciento dan mucha o bastante importancia a que en su dedicación puedan hacer amigos o a que estén acompañado por amigos y compañeros, el 68 por ciento. Descubre además que el 67 por ciento de los jóvenes rechaza explícitamente colaborar en asociaciones o instituciones que «tengan simpatías políticas». Lo significativo de este dato es que este porcentaje es superior entre los jóvenes que colaboran en actividades de ayuda a los demás (EDIS, 1995, p. 107).

Estos datos coinciden, en gran medida, con los que aportan los estudios cualitativos. Los resultados obtenidos en diversas investigaciones sobre las motivaciones de los voluntarios jóvenes que colaboran en asociaciones no lucrativas, así lo confirman. En un estudio realizado sobre los voluntarios de Cruz Roja pudimos constatar la existencia de tres grupos de explicaciones que muestran la pluralidad de 


\begin{tabular}{|l|c|c|c|c|}
\hline & $\begin{array}{c}\text { Ninguna/ } \\
\text { poca }\end{array}$ & $\begin{array}{c}\text { Mucha/ } \\
\text { bastante }\end{array}$ & Ns/Nc & $\begin{array}{c}\text { Media } \\
(\mathbf{1 - 4})\end{array}$ \\
\hline Que sirva para mi actividad profesional & 50 & 45 & 5 & 2,39 \\
\hline Que haga amigos & 23 & 74 & 3 & 2,84 \\
\hline Que vea los frutos de la colaboración & 20 & 76 & 4 & 3,08 \\
\hline Que la organización no tenga simpatías políticas & 28 & 67 & 5 & 2,97 \\
\hline Qué me beneficie personalmente & 22 & 75 & 3 & 2,97 \\
\hline Que este acompañado de amigos y compañeros & 28 & 68 & 4 & 2,81 \\
\hline Que no sea un compromiso estable & 34 & 59 & 7 & 2,76 \\
\hline Lo religioso que uno sea influye en ayudar & 52 & 46 & 2 & 2,34 \\
\hline Colaborar con ONG, s es un buen modo de llenar el ocio & 46 & 51 & 3 & 2,50 \\
\hline Para colaborar hace falta buena formación & 56 & 41 & 3 & 2,33 \\
\hline No se colabora más porque nos se presentan oportunidades & 38 & 58 & 4 & 2,56 \\
\hline
\end{tabular}

Tabla 6. Importancia de las siguientes condiciones y nivel de acuerdo con las siguientes afirmaciones sobre la colaboración en actividades de ayuda a los demás. Año 1995 (jóvenes 15-29 años).

Escala: 1 ninguna, 2 poca, 3 mucha, 4 bastante.

Fuente: EDIS, La solidaridad de la juventud, 1995: 107.

orientaciones y opciones de su compromiso o implicación, no obstante, pudimos ver también que en todos ellos predominaba un componente de satisfacción y realización personal.

Un primer grupo de jóvenes admitía que decidieron hacerse voluntarios porque deseaban «ayudar a personas con problemas» pero de sus discursos y explicaciones se deducía que su impulso o su motivación profunda respondía a la necesidad de satisfacer necesidades expresivas: "conocer y relacionarse con otras personas», «tener nuevos amigos», «realizar actividades y tareas divertidas»...

Otro grupo vinculaba su decisión al deseo de ayudar a «personas necesitadas», pero confesaba también que en su incorporación habían influido «razones prácticas»: «adquirir experiencia laboral», «conseguir méritos para poder ampliar su curriculum vitae», etc.

Un tercer grupo, más reducido que los anteriores, admitía que decidieron hacerse voluntarios porque «deseaban contribuir al bienestar de otras personas», porque se sentían, urgidos a cumplir con lo que entendían era el «deber de ayudar a personas que lo necesitaban».
Los resultados obtenidos en nuestro estudio corroboran y coinciden con los aportados por EDIS y por otros estudiosos del voluntariado. Helena Béjar, analiza el lenguaje de los voluntarios de las organizaciones de acción social y comprueba que los voluntarios jóvenes justifican su incorporación a las asociaciones no por una exigencia derivada de un imperativo moral, sino por los beneficios que obtienen con dicha participación (Béjar, 2001). Del mismo modo, Zurdo constata que de los tres ejes básicos en los que gravita el proceso motivacional de los voluntarios/as - el moral, el social o comunitarista y el individualista (satisfacción de las propias necesidades afectivas o instrumentales) - es éste último el que predomina y se impone en los voluntarios jóvenes (Zurdo, 2003).

2.2. Disposiciones y orientaciones de la participación asociativa de los jóvenes españoles Los estudios sobre las motivaciones de los voluntarios jóvenes descubren las razones de su decisión pero no se fijan en la concepción de su participación ni precisan el modo en que 
plantean su compromiso. Este vacío nos animó a estudiar y analizar el comportamiento de los jóvenes que colaboran en un tipo representativo de asociaciones: las Organizaciones No Gubernamentales de Desarrollo (ONGD). Nuestro objetivo era, en este caso, identificar las características de su participación, los planteamientos y la orientación que definía su compromiso. Guiados por estas premisas, identificamos y diferenciamos tres variantes o tres concepciones de la ayuda que aparecían asociadas a tres modos de entender y de asumir su participación.

Comprobamos que un grupo de jóvenes participaba en un tipo de asociaciones dedicadas a la asistencia a personas y a colectivos que viven en países alejados. Estos jóvenes concebían su dedicación como una acción altruista y relacionaban su compromiso con prestar un servicio en un programa concreto o con la colaboración en alguna de las actividades que la asociación realizaba en su propio entorno social (recaudar dinero, captar socios y voluntarios, ayudar en la gestión de la asociación, etc.) Estas tareas las asumían y realizaban «cuando podían», cuando no estaban ocupados en otras labores consideradas por ellos como prioritarias (estudio, clases).

Observamos también que otro grupo prefería participar en asociaciones dedicadas a la ayuda humanitaria y a la realización de grandes proyectos de desarrollo en lugares alejados de su entorno social. Admitían que la dedicación a la asociación les suponía un coste de tiempo, pero la afrontaban en períodos y en momentos que no interrumpían sus estudios (vacaciones).

Tanto los voluntarios que participaban en tareas o programas dentro de nuestra sociedad como los que lo hacían en proyectos de desarrollo en países lejano no atribuían un alcance «político» a su compromiso; asumían que las ONGD tenían que ser «apolíticas» y seguían un patrón de participación centrado en el desarrollo de tareas, definido por la participación en actividades predeterminadas.

Apreciamos, así mismo, la presencia de un tercer grupo de jóvenes que optaban por implicarse en asociaciones dedicadas a la realización de proyectos de desarrollo y a la sensibilización de nuestra sociedad con los problemas y las carencias existentes en los países empo- brecidos (educación para el desarrollo). A diferencia de los anteriores éstos pensaban que el cometido de las asociaciones y de su participación «no debía reducirse a la realización de tareas asistenciales», sino que debía «contribuir al cambio de las sociedades, al logro de la justicia social y a la superación de las desigualdades». Entendían, por ello, que su compromiso y el de las ONGD tenían un alcance y una proyección políticos. Los planteamientos de este grupo diferían claramente de los anteriores, pero eran minoritarios dentro del conjunto de los jóvenes entrevistados.

\section{Conclusiones: ambivalencia y paradojas de la participación asociativa de los jóvenes españoles}

Los resultados de estos análisis descubren y reflejan las actitudes y las disposiciones que caracterizan a la participación asociativa de los jóvenes españoles; permiten afrontar y esclarecer algunas de las cuestiones planteadas al inicio del artículo sobre la trayectoria y los cambios de su compromiso asociativo, y sirven, además, para proponer una explicación sobre su implicación en asociaciones altruistas.

La primera evidencia que se desprende de los datos resaltados anteriormente es la de la permanencia, con pequeñas variaciones, durante las últimas cuatro décadas, de las tasas de participación asociativa de los jóvenes españoles. La continuidad y la estabilidad de las tasas de participación contrastan, no obstante, con el extraordinario dinamismo experimentado durante los últimos años en nuestro país por las asociaciones. Algunos analistas no dudan en atribuir ese crecimiento a la incorporación de los jóvenes en asociaciones altruistas (Mota, 1999, p. 45; Subirats, 1999, p. 30). Los datos de las encuestas no corroboran, sin embargo, esta tesis.

La expansión de las asociaciones no lucrativas y la implicación de los jóvenes en ellas se explica por otras razones. El crecimiento de las entidades del Tercer Sector en nuestro país se produce, tal como muestran distintos autores, por la confluencia de distintos factores: el cambio de las estructuras familiares (Ariño y Cuco, 2001), la emergencia de «nuevos riesgos sociales» (Marbán, 2010, p. 163), la reestructuración del Estado de Bienestar y el redimensionamiento de los servicios sociales (Rodrí- 
guez Cabrero, 2003). Dentro de estas entidades participan un número significativo de jóvenes, pero tal como constata Zurdo, «el conjunto de las asociaciones del Tercer Sector se ha «edificado», fundamentalmente, sobre una nueva generación de técnicos asalariados, y no tanto sobre una nueva «marea» de jóvenes participantes» (Zurdo, 2003, pp. 151-154).

La invariabilidad de las tasas de participación no implica, sin embargo, que las asociaciones en las que participan, los motivos y el modo en que se incorporan a ellas sigan siendo similares a las del pasado. Los datos empíricos confirman su distanciamiento de las asociaciones políticas y religiosas, sus preferencias por las asociaciones de corte expresivo, lúdico y festivo. Esta opción y, sobre todo, las nuevas disposiciones que adoptan en su implicación asociativa pueden atribuirse a sus preferencias valorativas. Los estudios sobre los valores de los jóvenes españoles descubren que sitúan en último lugar lo que tiene que ver con lo holístico, con lo ideológico: la política y la religión (Ariño, 2007, pp. 61-65; Elzo, 2009, p. 39).

Las inclinaciones que guían a los jóvenes españoles, sus opciones valorativas, además de distanciarles de las asociaciones políticas y religiosas, explican, en gran medida, los motivos que les impulsan a comprometerse en las asociaciones voluntarias. Los jóvenes españoles deciden hacerse voluntarios no por una determinación moral impersonal producto del sentido del deber, sino por una elección emotiva o utilitarista, porque les enriquece personalmente, porque les beneficia en un sentido práctico. Sólo un reducido grupo de jóvenes admiten que su opción surge por un sentimiento de indignación y por el deseo de transformar la realidad social (Tejerina, 2010, p. 72)

Este cambio ha ido acompañado además de un cambio en la orientación y en el modo en que los jóvenes asumen su compromiso asociativo. Hasta hace unas décadas la participación social se inspiraba en modelos que exigían una pertenencia y una identificación intensas con la organización. Hoy esos referentes y disposiciones no predominan en los jóvenes que se incorporan y participan en las asociaciones Una gran parte de los voluntarios jóvenes no «militan» en las asociaciones; se limitan a colaborar en alguna de las actividades que realiza la asociación; sus compromisos son a corto plazo, su dedicación es a tiempo parcial y no está socialmente estructurada sino individualmente orientada (Alonso, 1999, p. 14).

Esas opciones explican o permiten comprender su alejamiento de las asociaciones reivindicativas (movimientos sociales) y su neutralidad política. La mayoría participan no con la intención de transformar el mundo o la sociedad sino con el deseo de paliar las carencias y de prevenir la vulnerabilidad de colectivos y de personas que viven en situaciones de riesgo. Sólo uno un grupo minoritario y escasamente representativo reivindica el carácter «político» de su compromiso y de sus acciones.

Las preferencias que manifiestan en sus opciones asociativas (expresivas e instrumentales), el modo en que asumen su participación y el sesgo «apolítico» que adoptan en su participación nos hace dudar por ello del alcance político de la participación asociativa seguida por las nuevas generaciones. El talante y la orientación participativa de los jóvenes españoles más que una ciudadanía asociativa renovadora de la participación democrática reflejan una retirada de la política. Esta constatación no supone negar el alcance político de su participación, por supuesto que lo tiene, pero no contiene, tal como advierte Antonio Ariño, un proyecto político (Ariño, 2004, p. 100).

La delimitación de estos aspectos permite apreciar la ambivalencia y las contradicciones del compromiso asociativo de los jóvenes españoles. Los datos de las encuestas revelan, por un lado, la invariabilidad de las tasas de participación asociativa, pero descubren, por otro, el cambio de sus preferencias, de sus motivaciones y de sus disposiciones asociativas; confirman el incremento del número y del tamaño de las asociaciones, pero muestran también que ese incremento no se ha debido a la mayor implicación de los jóvenes ni tampoco ha supuesto un estímulo o un acicate para su compromiso o implicación social; confirman también la coexistencia de actitudes y de planteamientos en los que se entremezclan y combinan motivaciones y opciones diversas.

Estas constantes no se dan al margen de las tendencias y de los rasgos que definen o caracterizan a nuestra sociedad. Son, por el contrario, su reflejo y proyección. Los valores y las pautas que definen el compromiso de los jóvenes están en consonancia con la cultura domi- 
nante en nuestra sociedad; proyectan y expresan el predominio de una lógica conjuntiva y sintética propia y representativa de la época en que vivimos. Todo ello explica y permite comprender la ambigüedad y las paradojas de la participación asociativa de los jóvenes españoles.

\section{Referencias bibliográficas}

Alonso, L. E. (1999). La juventud en el tercer sector: redefinición del bienestar redefinición de la ciudadanía. Estudios de Juventud, 45, 9 -20.

Alonso, L. E. (2007). La crisis de la ciudadanía laboral. Madrid: Anthropos.

Ariño, A. (2004). Asociacionismo, ciudadanía y bienestar social. Papers, 74, 85-110.

Ariño, A. (dir.) (2007). Asociacionismo y voluntariado en España. Una perspectiva general. Valencia: Tirant lo Blanch.

Ariño, A. y Cuco, J. (2001). Las organizaciones solidarias. Revista Internacional de Sociología. 29, 7-39.

Ariño, A. y Llopis, R. (2003). Asociacionismo heterogéneo, voluntariado diverso. Revista Estudios de Juventud. ( Monografía: Jóvenes, Constitución y cultura democrática). Madrid: INJUVE.

Béjar, H. (2001). El mal samaritano. El altruismo en tiempos del escepticismo. Barcelona: Anagrama.

Benedicto, J. (2009). Los jóvenes frente a la política: ¿desenganchados, escépticos, alternativos o las tres cosas a la vez? En A. Ruíz (ed.), Jóvenes y compromiso ciudadano (pp. 59-88). Madrid: Pablo Iglesias.

Benedicto, J. y Luque, E. (2006). ¿Jóvenes despolitizados? Visiones y condiciones de la ciudadanía en tiempos difíciles. Panorama Social, 3, 108-119.

Callejo González, J. y Izquieta, J.L. (1996). Los nuevos voluntarios: entre el individualismo y la solidaridad. Valladolid: Diputación.

Comas, D. (2009). La participación política de los jóvenes en la España contemporánea. En J. Tezanos (ed.), Juventud y exclusión social (pp. 369-390). Madrid: Sistema.

EDIS. (1995). La solidaridad de la juventud. Madrid: INJUVE.

Elzo, J. (2009). Jóvenes en la sociedad del bienestar. En A. Ruíz Miguel (ed.), Jóvenes y compromiso ciudadano (pp. 31-58). Madrid: Fundación Pablo Iglesias.

Instituto de la Juventud. (febrero de 1978). III Encuesta Nacional de Juventud. Revista Cuadernos de Documentación, 1, Madrid: Instituto de la Juventud.

Izquieta, J.L. (2011). Voluntariado y Tercer Sector. Cultura, participación cívica y organizaciones solidarias. Madrid: Tecnos.

Izquieta, J.L. y Callejo, J.J. (2004). Las organizaciones de ayuda humanitaria y de cooperación al desarrollo. Revista Española de Investigaciones Sociológicas, 105, 195-206.

Marbán, V. (2010). El Tercer Sector en España. En Antonio Gutiérrez (ed.), Tercer Sector e Intervención Social (pp. 161-191). Madrid: UNED.

Mota, F. (1999). La realidad asociativa en España. En J. Subirats (ed.), ¿Existe sociedad civil en España? (pp. 42-65). Madrid: Fundación Encuentro.

Prieto Lacaci, R. (1985). La participación social y politica de los jóvenes. Madrid: INJUVE.

Prieto Lacaci, R. (1998). Tendencias del asociacionismo juvenil en los años 90. Madrid: INJUVE

Rodríguez Cabrero, G. (coord.) (2003). Las entidades voluntarias de acción social en España. Madrid: FOESSA.

Subirats, J. (1999). ¿Existe sociedad civil en España? Madrid: Fundación Encuentro.

Tejerina, B. (2010). La sociedad imaginada. Movimientos sociales y cambio cultural en España. Madrid: Trotta.

Torcal, M. y Montero, J. (2006). Political disafection in contemporary democracies: social capital, institutions, and politics. Londres: Routledge

Zurdo, A. (2003). Voluntariado y estructura social: funciones y límites. En G. Rodríguez Cabrero (ed.), Las entidades voluntarias de acción social en España (pp. 213-286). Madrid: FOESSA. 